\title{
Recommendations of the Polish Ophthalmological Society for contact-lens wearers in the COVID-19 era*
}

\author{
Jacek P. Szaflik', Andrzej Horban'², Joanna Przybek-Skrzypecka', Alina Bakunowicz-tazarczyk ${ }^{3}$, Dariusz Dobrowolski ${ }^{4}$, \\ Iwona Grabska-Liberek ${ }^{5}$, Justyna Izdebska' ${ }^{1}$ Jakub Kałużny ${ }^{6}$, Jerzy Mackiewicz, ${ }^{7}$, Marta Misiuk-Hojło9, \\ Ewa Mrukwa-Kominek ${ }^{10}$, Bożena Romanowska-Dixon ${ }^{11}$, Marcin Stopa ${ }^{12}$ \\ 'Department of Ophthalmology, Medical University of Warsaw, SPKSO Ophthalmic University Hospital, Warsaw, Poland \\ ${ }^{2}$ Clinic of Infectious Diseases in Adults, Medical University of Warsaw, Warsaw, Poland \\ ${ }^{3}$ Department of Pediatric Ophthalmology with Strabismus Treatment Centre, Medical University of Bialystok, Bialystok, Poland \\ ${ }^{4}$ Chair and Clinical Department of Ophthalmology, School of Medicine, Dentistry Division in Zabrze, Medical University of Silesia, Poland \\ ${ }^{5}$ Department of Ophthalmology, Centre of Postgraduate Medical Education, Warsaw, Poland \\ ${ }^{6}$ Department of Sense Organ Research, Collegium Medicum in Bydgoszcz, Nicolaus Copernicus University in Toruń, Poland \\ ${ }^{7}$ Department of Retina and Vitreous Humour Surgery, Medical University of Lublin, Lublin, Poland \\ 8Independent Public Teaching Hospital No. 1 in Lublin, Poland \\ ${ }^{9}$ Department and Clinic of Ophthalmology, Wroclaw Medical University, Wroclaw, Poland \\ ${ }^{10}$ Department of Ophthalmology, Medical University of Silesia, Katowice, Poland \\ "Department of Ophthalmology and Ocular Oncology, University Hospital in Krakow, Poland \\ ${ }^{12}$ Department of Ophthalmology, Chair of Ophthalmology and Optometry, Heliodor Swiecicki University Hospital, Poznan University of Medical \\ Sciences, Poznan, Poland
}

KEY WORDS: contact lenses, COVID-19, coronavirus SARS-CoV-2.

COVID-19 is a disease caused by the SARS-CoV-2 coronavirus. The pathogen spreads mainly through the droplet pathway. Breathing, coughing and sneezing of an infected person leads to the formation of an aerosol, inhalation of which leads to transmission of the infection. In addition, the excreted SARS-CoV-2 virus particles can cover various objects in the environment of the infected person, and the transmission of the virus particles from hands to the mucosa is an additional route of infection [1]. The study presented by N. van Doremalen indicated that the period of stability of the SARS-CoV-2 virus in the form of an aerosol is up to 3 hours, and on objects, depend- ing on the material of which they are made of, up to 4 hours (copper), 24 hours (cardboard paper) up to $80 \mathrm{~h}$ (plastic) [2].

Therefore, there is a theoretical risk of transmitting the SARS-CoV-2 virus to the conjunctiva during application of contact lenses. It is worth noting, however, that there is no scientific evidence for the role of the use of contact lenses in the transmission of infection, and in the opinion of experts of the Polish Ophthalmological Society it may highly probably be regarded as marginal.

At the same time, it should be stated that glasses can be an additional mechanical protective barrier against aerosol

\footnotetext{
${ }^{\star}$ Guidelines of scientific societies and associations (including the Polish Ophthalmological Society) do not constitute binding laws and do not determine the only correct procedures; they are only an opinion of a group of experts from a given field. The opinion reflects the current state of knowledge based on available scientific research results.

The guidelines do not exempt healthcare workers from personal liability with regard to making the correct decisions for individual patients. Personal responsibility for the used therapeutic methods rests with all individuals who practise medicine. It should be based on thorough knowledge and practical skills, while observing necessary safety measures with regard to oneself and the patient.

Readers of this paper are obliged to make themselves familiar with current information on the presented treatments and pharmacotherapies with special attention paid to manufacturers' information on doses, time, and administration as well as side effects of the used drugs.

The publishers and editors of the paper shall not be responsible for any damages that could in any way be connected to the contents of this paper.
} 
and the decision on how to correct vision should be made on individual basis. This position assumes that patients will comply with the routine hygiene regime applicable to all contact lens users [3].

1. Before putting on and removing contact lenses, the patient should wash their hands using running water and a detergent (e.g. soap). Hands should be wiped dry with a disposable paper towel.

2. In addition, during an epidemic, before washing hands, disinfecting the outer surface of the lens container and hands with $70 \%$ ethyl alcohol or another antiviral disinfectant could be considered.

3. The patient should change the fluid for storage and disinfection of contact lenses every day.
4. People wearing contact lenses should avoid the habit of touching or rubbing the eyes.

5. If this does not affect the comfort of use and vision, a change to one-day contact lenses may be suggested to the patient.

6. If the patient observes reddening of eyes while using contact lenses, they should be removed immediately and the patient should be seen by an ophthalmologist.

The document was consulted with and accepted by the Chief Sanitary Inspectorate.

Status as of 3.05.2020.

\section{DISCLOSURE}

The authors declare no conflict of interest.

\section{References}

1. Li Q, Guan X, Wu P, et al. Early transmission dynamics in Wuhan, China, of novel coronavirus-infected pneumonia. N Engl J Med 2020; 382: 1199-1207.

2. van Doremalen N, Bushmaker T, Morris DH, et al. Aerosol and surface stability of SARS-CoV-2 as compared with SARS-CoV-1. N Engl J Med 2020; 382: 1564-1567.

3. Bui TH, Cavanagh HD, Robertson DM, et al. Patient compliance during contact lens wear: perceptions, awareness, and behavior. Eye Contact Lens 2010; 36: 334-339. 